\title{
The Role of Type I IFNs in Influenza: Antiviral Superheroes or Immunopathogenic Villains?
}

\author{
Wenxin $\mathrm{Wu}^{\mathrm{a}}$ Jordan P. Metcalf ${ }^{\mathrm{a}-\mathrm{c}}$ \\ aDepartment of Medicine, Pulmonary, Critical Care and Sleep Medicine, The University of Oklahoma Health Sciences \\ Center, Oklahoma City, OK, USA; ${ }^{b}$ Department of Microbiology and Immunology, The University of Oklahoma \\ Health Sciences Center, Oklahoma City, OK, USA; 'Pulmonary Section, Medicine Service, Veterans Affairs Medical \\ Center, Oklahoma City, OK, USA
}

\author{
Keywords \\ Interferon · Influenza - Viral infection · Innate immunity . \\ Cytokines
}

\begin{abstract}
The important role of interferons (IFNs) in antiviral innate immune defense is well established. Although recombinant IFN- $a$ was approved for cancer and chronic viral infection treatment by regulatory agencies in many countries starting in 1986, no IFNs are approved for treatment of influenza A virus (IAV) infection. This is partially due to the complex effects of IFNs in acute influenza infection. IAV attacks the human respiratory system and causes significant morbidity and mortality globally. During influenza infection, depending on the strain of IAV and the individual host, type I IFNs can have protective antiviral effects or can contribute to immunopathology. In the context of virus infection, the immune system has complicated mechanisms regulating the expression and effects of type I IFN to maximize the antiviral response by both activating and enhancing beneficial innate cell function, while limiting immunopathological responses that lead
\end{abstract}

to exaggerated tissue damage. In this review, we summarize the complicated, but important, role of type I IFNs in influenza infections. This includes both protective and harmful effects of these important cytokines during infection.

(C) 2020 The Author(s)

Published by S. Karger AG, Basel

\section{Introduction}

Influenza A virus (IAV) is a member of the family Orthomyxoviridae and is an enveloped negative-stranded RNA virus that causes significant morbidity and mortality around the globe. The epithelial cells of the upper respiratory tract are the primary targets of IAV after overcoming local defenses including sialic acid-containing mucous in the epithelial lining fluid. While virus replicates in the epithelial cells, the virus also spreads to nonpermissive immune cells, such as macrophages and dendritic cells (DCs), in the respiratory tract of the lung [1, 2]. In these infected cells, the virus is recognized by 3 families of pattern recognition receptors (PRRs): Toll-like receptors (TLRs), RIG-I like helicases (RLRs) and nucleo-

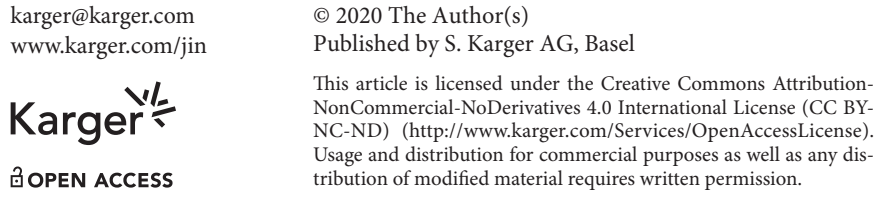

Wenxin $\mathrm{Wu}$

Department of Medicine

The University of Oklahoma Health Science Center

800 N. Research Pkwy, Room 425, Oklahoma City, OK 73104 (USA)

wenxin-wu@ouhsc.edu 


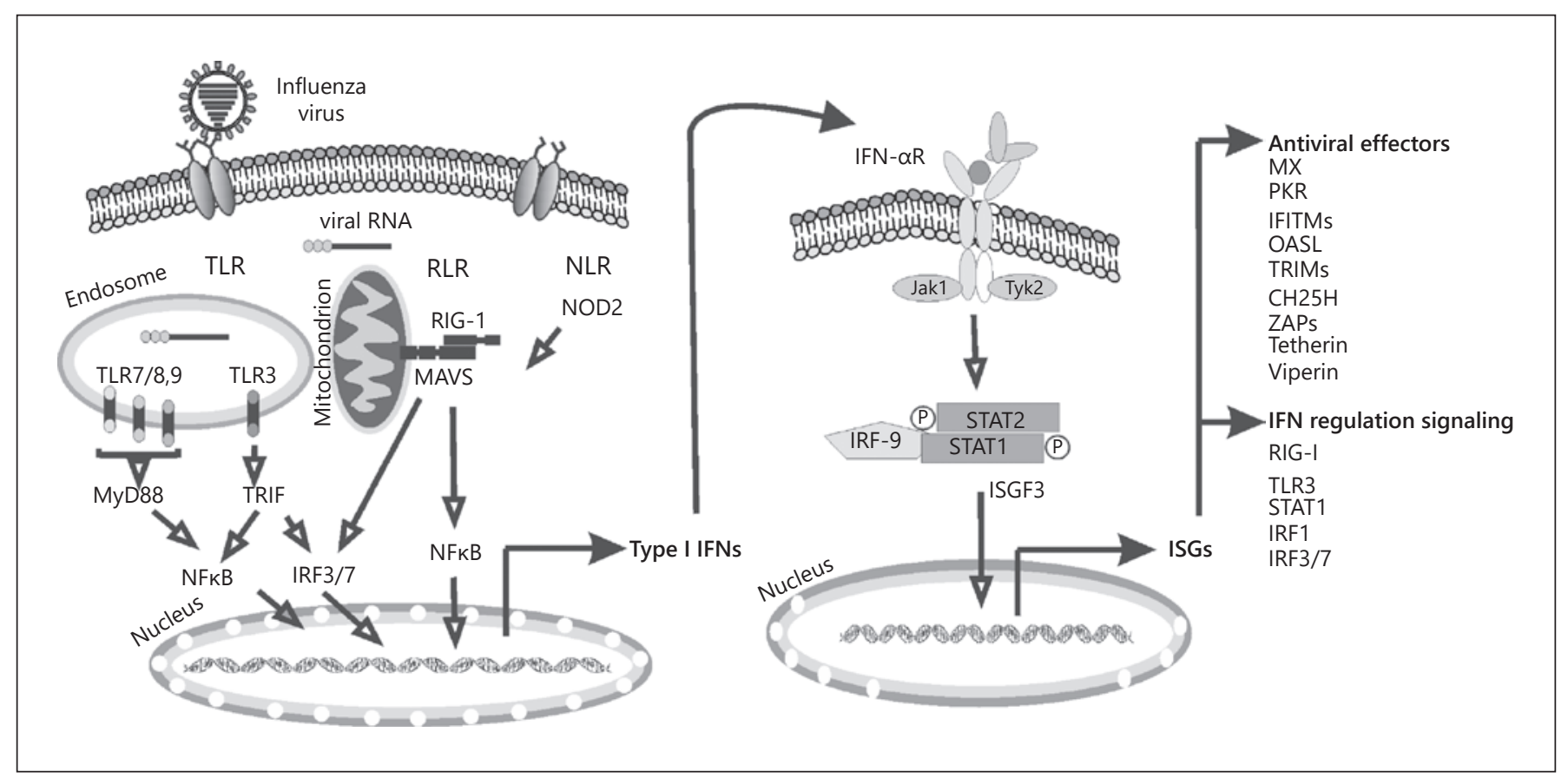

Fig. 1. Induction of type I IFNs and ISGs by influenza virus. Innate immune cells, such as macrophages and lung epithelial cells, produce type I IFNs after sensing IAV genomic RNA using various PRRs. In infected and neighboring cells, type I IFN signaling activates the JAK-STAT pathway, leading to transcription of ISGs, the products of which initiate intracellular antiviral effectors that lim- it the spread of the viruses. IFN, interferon; ISG, IFN-stimulated gene; PRR, pattern recognition receptor; JAK, Janus kinase; STAT, signal transducer and activator of transcription; TLR, Toll-like receptor; RLR, RIG-I like helicase; NLR, nucleotide-binding domain and leucine-rich-repeat-containing protein. tide-binding domain and leucine-rich-repeat-containing proteins (NLRs). The recognition and binding of virusspecific nucleic acids by PRRs induce production and secretion of interferons (IFNs) and proinflammatory cytokines, which are critical components of the antiviral response in mammals. IFNs are the major cytokines expressed during the host response with antiviral, antiproliferative, and immunomodulatory effects to viral or bacterial infection. Besides their role in restricting infection, IFNs also have been involved in both cancer immunosurveillance and autoimmunity $[3,4]$.

IFNs can be produced by virtually all nucleated cells of vertebrates and are classified as type I, type II, or type III IFN according to their genetic, structural and functional characteristics and specific receptors on the cell surface [5]. In 1957, Lindenmann et al. [6] discovered a substance that protected cells from IAV infection - they called it interferon [6]. In humans and mice, type I IFNs are composed of 19 IFN proteins: 14 IFN- $\alpha$ subtypes (IFN- $\alpha 1$ to $\alpha 14)$, IFN- $\omega$, IFN- $\varepsilon$, IFN- $\tau$, IFN- $\kappa$, and IFN- $\beta$. IFN- $\alpha$ and IFN- $\beta$ can be expressed by nearly every cell type $[7,8]$. The type II IFN family is represented by a single gene product, IFN- $\gamma$, and is mainly produced by $\mathrm{T}$ lymphocytes and natural killer (NK) cells $[9,10]$. Type III IFNs comprise 4 subtypes, IFN- $\lambda 1$, IFN- $\lambda 2$, IFN- $\lambda 3$, and IFN- $\lambda 4$, and are also expressed in multiple cell types [11, $12]$.

IAV are strong inducers of all types of IFNs at various stages of infection [13]. In this review, we will focus on the roles of type I IFNs induced during IAV infection because innate and adaptive immune responses to IAV in mammals are greatly influenced by type I IFNs.

\section{Type I IFN Inhibition of IAV Replication}

Type I IFNs, upon IAV infection, stimulate the expression of hundreds of genes that are altogether known as interferon-stimulated genes (ISGs) that act to eliminate the virus and prevent its spread by promoting an antiviral state in nearby cells (Fig. 1). ISGs all display the same characteristic structure of helical cytokines with a bundle of 4 a helices organized in an "up-up-down-down" configuration and also contain an additional separate $\alpha$ helix [14]. 
As secreted proteins, type I IFNs act as intercellular messengers and exert potent biological effects at extremely low concentrations through the type 1 IFN- $\alpha / \beta$ receptor (IFNAR), a cell surface transmembrane receptor. IFNAR is composed of 2 subunits - IFNAR1 and IFNAR2. Typically, IFNARs are endocytosed and activate their associated tyrosine kinases, Tyk2 and Jak1 [15], followed by binding of type I IFNs. Jak1 activates signal transducer and activator of transcription (STAT) 1 by phosphorylation. This classical signaling cascade results in formation of IFN-stimulated gene factor 3 (ISGF3), a complex of phosphorylated STAT1 and STAT2 with IRF9. The activation of ISGF3 leads to the increased expression of over 100 ISGs, including 2',5'-oligoadenylate synthetase (OAS), Mx proteins, interferon-induced transmembrane protein 3 (IFITM3), and protein kinase R (PKR), inducing an antiviral state [16].

The role of type I IFNs in IAV pathogenesis is complex. For example, mice genetically deficient in type I IFN signaling had inefficient IAV clearance $[17,18]$. When given prophylactically, type I IFN reduces IAV replication and disease severity in both animals [19] and humans [20]. Engagement of the type I IFN response prior to infection may be a therapeutic strategy to control IAV infection in different animal models, but of course has limited practical applications [21,22].

Challenge experiments with 2 different strains of IAV revealed significantly reduced survival rates and enhanced virus titers in the lungs of IFN- $\beta$-deficient mice, demonstrating that IFN- $\beta$ contributes to innate immunity against IAV [18]. Since the STAT1 protein is required for signaling from type I IFN, STAT1-/- animals are 100-fold more sensitive to lethal A/WSN/33 (H1N1) IAV infection than are their wild-type (WT) counterparts [23]. Additionally, as shown by García-Sastre's group [23], the $\mathrm{LD}_{50}$ of IAV PR8 virus was 10 times lower in STAT1-/- mice than in WT mice. In vitro, WSN33 replicates to high titers in STAT1-/- or IFNAR-/- fibroblasts, while cells derived from WT animals are resistant to IAV infection [23]. However, these results did not directly translate into in vivo models using IFNAR-/- mice by other groups. X31 (H3N2) IAV titers in the lungs of IFNAR-deficient mice were not significantly different from WT controls, and IFNAR-/- and WT mice were comparably susceptible to X31 infection [24].

Excellent work from Crotta et al. [25] later explained the apparent contradictory results using STAT1-/- and IFNAR1-/- mice during IAV infection [25]. The difference appeared to be due to differing types of IFN effects in mouse lung epithelia. IAV were more pathogenic and

Type I IFNs: Double-Edged Sword? replicated to higher titers in the lungs of mice lacking both IFNAR and IFN- $\lambda$ receptor (IFNLR) than in mice with single IFN receptor defects. Using bone marrow chimeric mice with WT donors and IFNAR1/IFNLR double-deficient recipients, the lack of both type I and type III IFN signaling in the stromal compartment significantly increased the susceptibility to influenza infection. Specifically, when these chimeras were infected with IAV PR8, high susceptibility and mortality were found only in the group lacking IFN receptors on stromal cells and this correlated with higher viral titers [25]. This study demonstrates the overlapping, and potentially compensatory function of type I and type III IFNs in controlling IAV, as knockout of both receptors is required for increased susceptibility to IAV infection. As STAT1 activation is required for signaling through both receptors, STAT1 knockout inhibits function of both type I and type III IFN signaling pathways. Thus, results using the chimeric double knockouts clarify the confusion arising from earlier literature that reported that type I IFNs cannot alone account for the requirement for STAT1 signaling in protection against IAV infection.

Additional studies have revealed that unlike type I IFN receptors, expression of functional IFN- $\lambda$ receptor complexes are restricted to epithelial cells in the lung and intestinal tract. Therefore, type III IFN effects might be limited to combating virus replication at mucosal surfaces due to the limited expression of the IFN- $\lambda$ receptors [11]. Type I IFN signaling is more important in limiting the spread of systemic IAV infection due to its universal distribution in all types of cells. This paradigm of epithelial-exclusive action of type III IFN has recently been challenged as other cells such as neutrophils and DCs also respond to IFN- $\lambda$ [26]. On the other hand, recent work with IAV-infected mice [27] indicated that IFN- $\lambda$ is produced more quickly than type I IFN, suggesting that type III IFN plays a nonredundant role in suppressing early virus growth in the respiratory tract. IAV spreads more rapidly from the nasal cavity to the lungs in mice whose type III IFN system was defective. Infections in mice that lacked type III IFN were also more likely to spread to other animals. Furthermore, treating mice with type III IFN, but not type I IFN, gave their upper respiratory tract long-lasting protection against flu infections and prevented the spread of the virus [28]. Thus, type III IFN constitutes a first line of antiviral noninflammatory response while type I IFNs may act as a second line-responder and also enhance the production of inflammatory cytokines, in addition to the antiviral mediators. 


\section{Type I IFN-Induced Inflammation and Tissue Injury}

Apart from the above antiviral effects, evidence also hints at pathogenic roles for type I IFNs during viral infection. During chronic viral infection, in vivo studies have identified suppressive mechanisms involved in the harmful effects of type I IFNs. Chronic IFN signaling is associated with hyperimmune activation and disease progression in persistent infections [29]. There is a direct causal link between IFN signaling, immune activation, negative immune regulator expression, lymphoid tissue disorganization, and virus persistence [30]. Since influenza mainly causes acute infection, the details of detrimental effects in chronic viral infection will not be further discussed here.

In highly pathogenic IAV strain infection, such as the 1918 pandemic H1N1 and avian H5N1 strain, IAV causes pneumonia in humans with progression to lung failure and fatal outcome. Progressive primary viral pneumonia is commonly observed, with secondary bacterial pneumonia more prominent following the 1918 pandemic virus outbreak than in H5N1-infected individuals [31]. Recombinant IAV bearing the 1918 virus hemagglutinin (HA) and neuraminidase (NA) glycoproteins were reconstructed in the genetic background of the human H1N1strain (1918 HA/NA:Tx/91). Although the lung IFN- $\alpha$ levels were similar for both virus-infected groups, IFN- $\gamma$, TNF- $\alpha$, MIP-1 $\alpha$, and MIP-2 chemokines were detected at significantly higher levels in 1918 HA/NA:TX/91-infected mice than in seasonal TX/91-infected mice [32, 33]. Nonhuman primates infected with reconstructed 1918 pandemic virus demonstrated a dysregulated expression of the innate immune response, which may be a critical determinant of the severity and outcome of infection by the 1918 pandemic virus. The 1918 virus induced an antiviral response different and less protective than the response to a seasonal H1N1 strain [34]. 1918 virus infection induced much fewer IFN- $\alpha$ genes than conventional virus. IFN- $\beta$ mRNA was either not induced or was downregulated in all samples by 1918 virus. In regards to avian influenza virus, in vitro studies have also demonstrated robust induction of proinflammatory cytokines, in particular TNF- $\alpha$ and type I IFN, by $\mathrm{H} 5 \mathrm{~N} 1$ viruses compared to other human $\mathrm{H} 3 \mathrm{~N} 2$ and $\mathrm{H} 1 \mathrm{~N} 1$ viruses [35]. Other studies have highlighted the strong correlation between IL-6, IFN- $\alpha$, and TNF- $\alpha$ levels and the severity of disease symptoms. As in experimental influenza, symptoms and fever in natural acute influenza correlate with the release of IL-6 [36, 37].

In seasonal virus, many proinflammatory cytokines and chemokines are also induced downstream of IFNAR signaling which may cause an exaggerated inflammatory response and tissue injury. One early study found that local respiratory production of IFN- $\alpha$ in humans correlated with infection and illness severity [36]. A major determinant of this IFN- $\beta$ induced detrimental host response was found to be the potent apoptosis-inducing death receptor 5 (DR5), which functions as a receptor for tumor necrosis factor-related apoptosis-inducing ligand (TRAIL) in lung pneumocytes [38]. It was shown that severe IAV infection is associated with TRAIL-mediated apoptosis in epithelial injury upon viral pneumonia in mice and humans [39, 40] and that IFN- $\alpha / \beta$ can induce TRAIL expression by IA $V$-infected macrophages and pDCs $[39,41]$.

Later work by Davidson et al. [42] confirmed that excessive type I IFN signaling in response to acute IAV infection can result in uncontrolled inflammation [42]. This effect is likely related to the induction of epithelial cell death, secondary to the proapoptotic effects of type I IFN. More susceptible mouse strains produce markedly higher levels of IFN in response to influenza infection than resistant strains. High numbers of hyperreactive pDCs produced excessive IFN amounts sustained over time, which in turn caused uncontrolled inflammation and lung epithelial damage mediated by TRAIL-DR5 interactions [39]. The induction level of Fas ligand (FasL) gene expression in the lung has also been correlated with the severity of influenza infection and type I IFN is critical for induction of FasL protein expression in the lung [43]. Through this mechanism, excessive type I IFN has been implicated in lung injury and death in severe IAV infection.

Different populations may have specific responses to type I IFN. Infants and young children are known to have a higher risk for poor clinical outcomes after infection with IAV [44]. Studies found minimal viral load increases in the very young and no age association with existing antibody titer [45-47]. Instead, a far more striking age association was observed with subsets of cytokines, including type I IFNs [48]. Elevated levels of IFN- $\alpha 2$ in nasal lavage correlated with increased disease severity even after accounting for the factors associated with age [49]. Local airway immune responses are known to be critical determinants of infection kinetics and disease progression.

Postinfectious therapeutic administration is ineffective in animal models and may actually increase lethality despite a reduction in lung IAV titers [19]. A prolonged or exaggerated type I IFN response at later stages might worsen the inflammatory response in IAV pneumonia [39]. To prevent potent adverse effects from proinflammatory functions of IFNs, multiple negative regulatory mechanisms involve a diverse range of molecules that act 


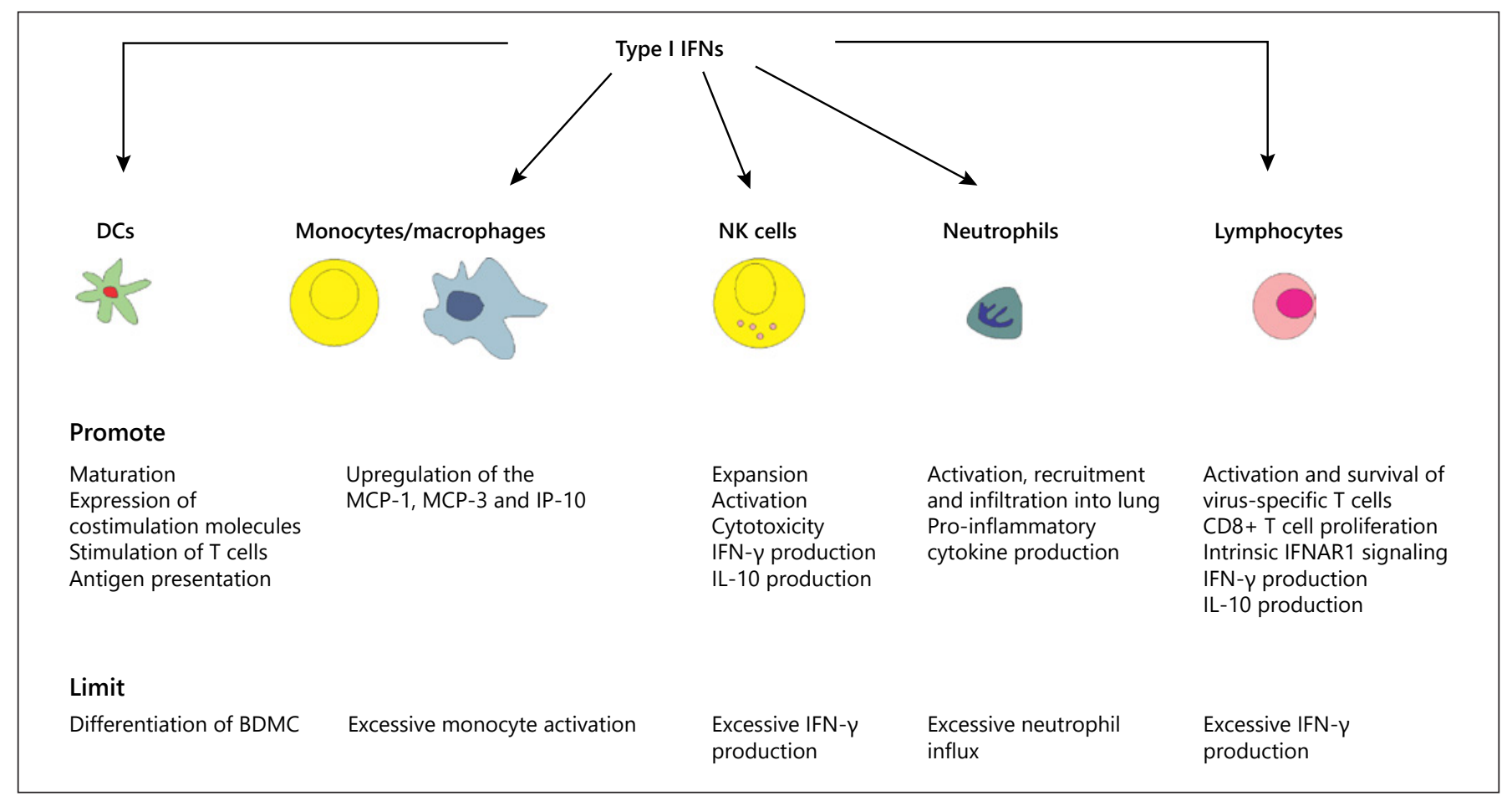

Fig. 2. Type I IFNs modulate immune cell functions during influenza virus infection. IFN, interferon; NK cell, natural killer cell; DC, dendritic cell; IFNAR, IFN- $\alpha / \beta$ receptor.

at all points throughout the IFN signaling pathway to control type I IFN production, signal transduction, and IFN-mediated transcription and translation (reviewed by Porritt and Hertzog [50]). Negative regulatory mechanisms act to calibrate the IFN response allowing virus clearance while maintaining homeostasis. Type I IFNs mediate the upregulation of expression of apoptosis-inducing proteins expressed by nonhematopoietic somatic cells mediating tissue damage. The same molecules, when induced in immune cells by IFNs, can contribute to immunosuppression in a similar manner to PDL1 and IL10. Therefore, type I IFNs can exaggerate or reduce inflammation and pathology depending on experimental conditions. Factors such as timing, signaling magnitude, cell source, and the individual IFN subspecies studied have to be considered when evaluating the effects of IFN induction on the host or target cell.

\section{Immunoregulatory Functions}

In contrast to their proinflammatory effects, a growing amount of evidence suggests that type I IFNs have immunoregulatory functions that are critical for dampening immunopathogenic mechanisms and minimizing collateral damage from the infection [51]. They contribute to key modulation of antiviral functions in DCs, monocytes, neutrophils, NK cells, and T cells (Fig. 2). Proinflammatory cytokines are positive mediators of local and systemic inflammation, induce fever, initiate tissue destruction, and modulate the adaptive immune response to IAV. Anti-inflammatory cytokines serve to reduce inflammation and promote healing. The net effect of an inflammatory response is determined by the balance between proinflammatory and anti-inflammatory cytokines. Both proand anti-inflammatory effects on airway cell populations have been reported after oral or systemic administration of IFN- $\alpha$ in mice and horses [52-55].

DCs are antigen-presenting cells (APCs) located at the portals of pathogen entry that are critical for activation of antiviral $\mathrm{T}$ cells [56]. Administration of type I IFNs to mouse and human DCs promotes DC maturation and enhances expression of costimulatory molecules and DC stimulation of T cells [57, 58]. Simmons et al. [59] showed that type I IFN drives a distinctive DC maturation program that enhances antigen presentation to $\mathrm{T}$ cells without a shutdown of antigen processing, allowing continued sampling of antigens for presentation [59]. This may 
be beneficial in the course of IAV infection, as some DCs may be exposed to IFNs before they encounter the virus and antigens expressed by the virus, and it may be important to avoid premature shutdown of antigen processing before DCs are exposed to pathogens. However, some results define an opposing role for type I IFN in IAV-infected DCs. It is reported that IFN- $\alpha \beta$ inhibits the in vitro differentiation of DCs from CD14+ precursors. Type I IFN inhibited the differentiation of hematopoietic progenitors in a way that leads to reduced bone marrow-derived dendritic cell (BMDC) production [60]. These conflicting results might be due to divergent effects of type I IFNs on DC activation depending upon the maturation state of the DC [61].

Type I IFN operates as a master regulator controlling which DC subset will present antigens during a viral infection. Both subsets of lung DCs, CD103+ DCs and CD $11 b^{\text {high }}$ DCs, become infected by IAV in vivo and migrate to the MLNs, but only CD103+ DCs support productive virus replication. The phenomenon originates from a difference in sensitivity of the 2 DC populations to type I IFN [62]. CD103+ DCs express low levels of IFNAR compared to other DC subtypes and are resistant to type I IFN. The attenuated IFNAR signaling by CD103+ DCs correlates with their permissiveness for internal viral replication and enhanced antigen presentation capacity for naive CD8+ T cells when compared to CD11b high DCs. This may be due to greater viral antigen availability in CD103+ DCs. The notion of migratory lung CD103+ DC being permissive to IAV viral replication and type I IFNs enhancing their capacity as APC to CD8+ T cells has been challenged by later studies showing (i) a lack of viral replication in this subset during in vivo infection [63] and (ii) that infection of DC is not required for antigen presentation [64]. CD103+ DCs acquire and transport viral antigens from the lung to the draining lymph nodes, where they are capable of both direct and cross-presentation of viral antigens. Nevertheless, Helft et al. [63] also observed that CD103+ DC resistance to infection correlates with an increased antiviral state in these cells that is dependent on the expression of the type I IFN receptor [63]. These results show that efficient cross-priming by migratory lung DCs is coupled to the acquisition of an antiviral state, which is dependent on the type I IFN signaling pathway. Interestingly, recent work has found that CD103+ DCs might rely on IFN- $\lambda$ signaling for optimal activation through the IFN- $\lambda$ receptor [65]. Thus, although antigen presentation by lung DCs is proportional to virus replication and is tightly constrained by type I IFN, optimal activation of DCs may, in contrast, require type III IFN.
After IAV infection, the virus triggers an initial release of inflammatory mediators resulting in acute pulmonary inflammation associated with the recruitment of inflammatory monocytes and neutrophils in infected lungs [31]. Flow cytometry and gene expression analysis involving isolated cell subpopulations from infected lungs showed that neutrophil influx largely accounted for the predictive transcriptional signature. Neutrophil reduction prevented host death from self-amplifying damaging inflammation [66]. Galani et al. [27] found that type I IFNs constituted major inducers of neutrophil infiltration and neutrophils were the main cells producing proinflammatory mediators in response to IAV infection [27]. Type I IFNs are involved in the neutrophil-driven proinflammatory cascade in the lung that is induced following IAV infection. In parallel, type I IFNs synergize with IFN- $\gamma$ to inhibit neutrophil infiltration and suppress neutrophil chemotactic chemokine/cytokine production in IAV infection [67]. In such cases, type I IFN signaling is necessary, but not sufficient, for preventing neutrophil recruitment into the lungs of IAV-infected mice. Type I IFNs promote upregulation of the MCP-1, MCP-3, and IP-10 chemokines, with amplification of the inflammatory/chemotactic signal and further recruitment of monocytes/macrophages and T lymphocytes to the site of infection [68]. New research reveals a novel IFN-dependent regulatory mechanism designed to prevent excessive immunopathology while preserving its antiviral functions. IFNAR1-/- mice develop significant defects in the infiltration of Ly6 $\mathrm{C}^{\text {hi }}$ monocytes in the lung after IAV infection. Ly6 $\mathrm{C}^{\text {hi }}$ monocytes of WT mice are the main producers of MCP-1 while the alternatively generated Ly $6 \mathrm{C}^{\text {int }}$ monocytes of IFNAR-/- mice mainly produce cytokines for neutrophil influx. As a consequence, IFNAR1-/- mice recruit more neutrophils after influenza infection than do WT mice [17]. The protective function of type I IFNs is associated with not only the recruitment of classical inflammatory Ly6 $\mathrm{C}^{\text {hi }}$ monocytes into IAV-infected lungs, but also the prevention of excessive monocyte activation by IFN- $\gamma$ [67]. Thus, it appears that type I IFN dictates homeostasis of hematopoietic stem cells by controlling neutrophil influx into, and monocyte activation at, the site of inflammation.

NK cells are large, granular, innate lymphoid cells that act as immune regulators through cytokine production and as cytotoxic effector cells. Their main functions during viral infection are the production of IFN- $\gamma$ and containing viral replication by killing infected cells early after influenza infection [69]. Type I IFN has been shown to play a notable role in IAV-mediated NK cell expansion and activation $[70,71]$. There is direct action of type I 
IFNs on NK cells, enhancing effective NK cell responses in the context of flu infection and facilitating activation of NK cell signaling pathways responsible for cytotoxic activity and cytokine production [72]. Arimori et al. [73] showed that IFNAR-/- mice exhibited impaired cytotoxic activity as well as an increased ability of NK and CD8+ T cells to produce IFN- $\gamma$ after infection with IAV. Therefore, type I IFN signaling plays a role not only in the upregulation of cytotoxicity but also in the downregulation of some effector mechanisms including IFN- $\gamma$ production by NK and CD8+ T cells via IL-10 production [73].

Type I IFNs are important in promoting the activation and survival of virus-specific $T$ cells and establishment of immune memory [74-76]. Type I IFNs exert potent costimulatory effects on CD8+ T cells, enhancing CD8+ T cell proliferation upon T-cell intrinsic IFNAR1 signaling [77]. Type I IFN plays a major role in the CD8+ T cell response to viral infection, and its effects are on both the APCs and the T cells. The phenotype of the T cells and the timing of IFN exposure are important, as IFN can inhibit proliferation or induce apoptosis under some circumstances yet be dramatically stimulatory under other conditions. Depending on their activation status, T cells can change their expression levels of IFNAR and their expression of signaling molecules downstream from the IFNAR. First, IFN upregulates MHC and costimulatory molecules. Second, IFN promotes apoptosis of preexisting memory $\mathrm{T}$ cells, which are rapidly phagocytozed by CD8 $a+$ DCs. Third, IFN directly promotes the proliferation of antigen (Ag)-specific CD8+ T cells at the beginning of the response. Fourth, IFN indirectly enables late arriving Ag-specific T cells to become immediate effectors but directly inhibits proliferation of these cells [77].

Clinical observations and rodent models of human disease have revealed that group 2 innate lymphoid cells (ILC2 cells) serve critical roles in allergic inflammatory responses such as asthma and lung inflammation induced by pathogens. Duerr et al. [78] observed increased ILC2 cells and more deregulated innate and adaptive type $2 \mathrm{im}$ munity in IFNAR-deficient mice infected with IAV than in infected WT mice, which demonstrated that type I IFNs were central regulators of ILC2-mediated adverse immune responses that can lead to tissue pathology [78].

\section{Distinct Effects of IFN- $\beta$}

IAV infection mostly induces a mixture of different IFN- $\alpha$ subtypes and IFN- $\beta$. For many years, type I IFNs were named as IFN- $\alpha / \beta$. However, many studies have re-

Type I IFNs: Double-Edged Sword? vealed that individual subtypes of type I IFN can have differing effects, despite signaling through the same receptor, leading to different antiviral function and biological effect.

During investigation of the activity of type I IFN subtype enhancement of DC differentiation, transcriptome analyses found that the expression level of 7 chemokines and several DC surface markers distinguished IFN DC subtypes, IFNa-DC and IFN $\beta$-DC [79]. Differences in antiviral activity were also found among the all type I IFN subtypes as measured by inhibition of lung virus replication [80].

Several groups demonstrated that there is a hierarchy of temporal expression within the type I IFN gene family $[81,82]$. IFN- $\beta$ is an early responder and plays an essential role in the efficient induction of all type I IFNs after infection of primary embryonic as well as primary adult fibroblasts with Sendai virus. Studies on primary fibroblasts of mice with a targeted deletion of the IFN- $\beta$ gene largely confirmed the view that IFN- $\beta$ serves as an immediate early IFN [83]. Production of IFN- $\beta$ does not require signaling through IFNAR $[84,85]$. In addition, binding of IFN- $\beta$ to IFNAR induces subsequent transcriptional responses leading to IFN- $\alpha$ expression. IFN- $\beta-/-$ mice are highly susceptible to vaccinia virus infection, in part because of a failure to mount an appropriate IFN- $\alpha$ response [86]. During influenza infection, respiratory epithelial cells are important sources of IFN- $\beta$ early while pDCs act later by subsequently releasing high amounts of IFN- $\alpha$ and IFN- $\beta$ [87].

In terms of gene expression regulation, both the promoters of IFN- $\alpha$ and IFN- $\beta$ genes have interferon regulatory factor (IRF) binding sites which allow the family of IRF transcription factors to drive the production of IFNs. However, the promoter of IFN- $\beta$ has other response elements, including sites for NF- $\kappa \mathrm{B}$ and AP-1 [88]. IFN- $\beta$ can be produced in more diverse circumstances compared to those that result in the production of IFN- $\alpha$ subtypes. This less restricted production of IFN- $\beta$ implies a function of IFN- $\beta$ that is different relative to other type I IFNs.

\section{Molecular Basis of Homeostasis Role of Type I IFNs}

How can type I IFNs play such a complicated role in generating a multitude of signals? The mechanisms may be due to variation in the structures of the 19 type I IFNs as well as differences in their receptors. Specifically, distribution of the receptors and binding affinity between 
the IFN and receptor may also play a role in differential IFN signaling responses.

Type I IFNs are recognized by and signal through the heterodimeric IFNAR comprised of IFNAR1 and IFNAR2. Most studies on the role of type I IFN signaling in regulating host susceptibility to IAV have been focused only on IFNAR1 deficiency (using IFNAR1-/- mice). However, the individual receptor subunits can bind IFN- $\beta$ or IFN- $\alpha$ 's independently of each other and induce distinct signaling. IFN- $\beta$ and IFN- $\alpha$ 's are known to have different affinities for IFNAR1 and IFNAR2 and to induce different gene expression profiles depending on their concentration and timing [50]. IFN- $\beta$ has a 20 - to 30 -fold higher affinity for either IFNAR1 or IFNAR2 compared to IFN- $\alpha 2$ [89]. The high-affinity properties of IFN- $\beta$ may explain the 40- to 60 -fold increase in cell proliferation activity of this IFN subtype relative to IFN- $\alpha 2$. An additional effect of this high receptor affinity relative to other type I IFN subspecies may explain why only IFN- $\beta$ induces the negative immune regulating factors IL-10 and programmed death-ligand 1 (PDL1) [90, 91], which suppress $\mathrm{T}$-cell responses contributing to viral clearance.

Pioneering work from Thomas et al. [92] revealed that differential IFN affinities capable of inducing diverse functional effects appears to be due to ligand discrimination through distinct receptor-binding chemistries, which dictate the respective stabilities of the receptor-ligand interactions [92]. Increased binding affinities toward IFNAR1 or IFNAR2 strongly enhance receptor downregulation. The suppressed innate immune effects of type I IFN required higher binding affinities to IFNAR. Later, de Weerd et al. [88] established that IFN- $\beta$ uniquely and specifically ligates to IFNAR1 in an IFNAR2-independent manner. The IFNAR1-IFN- $\beta$ complex transduced signals that modulated expression of a distinct set of genes independently of Jak-STAT pathways. IFNAR1IFN- $\beta$ signaling is pathologically relevant as lipopolysaccharide-induced sepsis was ameliorated in IFNAR1-/mice but not IFNAR2-/- mice [88].

Levin et al. $[93,94]$ showed that type I IFN variants or mutants induced 2 different patterns of gene expression based on IFN receptor affinity, receptor number, and concentration of IFN $[93,94]$. They referred to genes expressed in the first pattern as "robust" genes, with many of them related to antiviral activities, whereas genes expressed in the pattern, whose products have immunomodulatory and antiproliferative functions, are termed "tunable" genes. All IFNa bind the IFNAR1 receptor subunit with low affinity. Increasing the binding affinity enhanced the antiproliferative activity of IFNa2 [95].
Jaks et al. [96] found that the ISGF3 formation and antiviral activity correlated very well with the binding affinity of IFNs toward IFNAR2. In contrast, the affinity toward IFNAR1 played a key role for antiproliferative activity [96]. Recently, Shepardson et al. [97] demonstrated that despite some redundancies, IFNAR1 and IFNAR2 have distinct roles in regulating anti-IAV immunity. In contrast to IFNAR1-/- mice, IAV-infected IFNAR2-/mice displayed both increased and accelerated morbidity and mortality compared to WT mice [97]. Treatment of mice that have functional IFNAR2 but not IFNAR1 (IFNAR1-/- mice) with IFN- $\beta$ protected these mice from morbidity and increased their survival compared to IAVinfected WT littermates. However, IFNaA treatment of mice deficient in either IFNAR subunit had no effect on IAV-induced body weight loss when compared to their untreated littermates. Thus, unlike IFNAR1, IFNAR2 was sufficient to generate protection from lethal IAV infection when stimulated with IFN- $\beta$.

Together, the affinities and residence time of receptor binding, level of surface receptor expression, and the cell types that the receptors are located may determine the different responses among all type I IFN subtypes [98]. Multilayer feedback mechanisms are available to prevent detrimental outcomes of IFN receptor and downstream signaling activation.

\section{Summary}

IAV infection is a major cause of infectious morbidity and mortality worldwide [99]. Recent studies in mouse models have revealed a pivotal role for type I IFNs in host defense against IAV. They quickly activate downstream ISG signaling networks and restrict viral replication. Type I IFNs promote activation of innate immune cells, induce adaptive immunity, and regulate innate and adaptive responses. Many of the findings regarding IFN effects in mouse models have led to the discovery of parallel phenomena in humans. While they cannot fully replicate the human disease, mouse models have enabled researchers to use genetic strategies to decipher mechanisms that are critical for IFN responses to IAV in vivo. However, there are significant gaps in our knowledge of the role of type I IFNs in human influenza infection. Further studies are still needed to elucidate the role of type I IFNs in the host response to IAV in humans.

Type I IFNs must be tightly regulated to maximize viral clearance while inflicting minimal damage to host cells. It is especially challenging when dealing with influ- 
enza infection since mortality from IAV is strongly linked to secondary bacterial infections. Viral clearance must be accomplished with rapid resolution of inflammation caused by the primary viral infection, or facilitation of invasion by pathogens and secondary bacterial pneumonia will occur [100]. Understanding of type I IFN effects will provide important practical implications, including the possible use of immunosuppressive or anti-inflammatory measures in influenza therapy.

The use of biologic response modifiers in human disease has come of age for many illnesses including cancer, autoimmune diseases, and some viral infections. In order for type I IFNs or other IFNs to be used therapeutically in influenza infection, we must understand their mechanisms so that they can be used as antiviral superheroes, rapidly resolving viral infection, and not become immunopathogenic villains, worsening the effects of virus-mediated tissue injury.

\section{Acknowledgements}

The authors wish to thank Dr. Gillian M. Air (OUHSC, Oklahoma City, OK, USA) for critically reading the manuscript and for helpful comments.

\section{Disclosure Statement}

The authors declare no conflicts of interest.

\section{Funding Sources}

The work was partially supported by Oklahoma Shared Clinical and Translational Resource (OSCTR) pilot grants (grant number U54GM104938 to W.W.), the Merit Review Program of the Department of Veterans Affairs (grant number I01 BX001937 to J.P.M.), and the National Institute of General Medical Sciences (grant number 5P20GM103648 to J.P.M).

\section{Author Contributions}

Both W.W. and J.P.M. were involved in manuscript writing.

\section{References}

1 Manicassamy B, Manicassamy S, Belicha-Villanueva A, Pisanelli G, Pulendran B, GarcíaSastre A. Analysis of in vivo dynamics of influenza virus infection in mice using a GFP reporter virus. Proc Natl Acad Sci U S A. 2010; 107(25):11531-6.

2 Coccia EM, Severa M, Giacomini E, Monneron D, Remoli ME, Julkunen I, et al. Viral infection and Toll-like receptor agonists induce a differential expression of type I and lambda interferons in human plasmacytoid and monocyte-derived dendritic cells. Eur J Immunol. 2004;34(3):796-805.

3 Di Franco S, Turdo A, Todaro M, Stassi G. Role of type I and II interferons in colorectal cancer and melanoma. Front Immunol. 2017; 8:878.

4 Hall JC, Rosen A. Type I interferons: crucial participants in disease amplification in autoimmunity. Nat Rev Rheumatol. 2010;6(1):409.

5 Secombes CJ, Zou J. Evolution of interferons and interferon receptors. Front Immunol. 2017;8:209.

6 Lindenmann J, Burke DC, Isaacs A. Studies on the production, mode of action and properties of interferon. Br J Exp Pathol. 1957; 38(5):551-62.

7 Swiecki M, Colonna M. Type I interferons: diversity of sources, production pathways and effects on immune responses. Curr Opin Virol. 2011;1(6):463-75.

8 Pestka S, Krause CD, Walter MR. Interferons, interferon-like cytokines, and their receptors. Immunol Rev. 2004;202;8-32.
9 Klein JR, Raulet DH, Pasternack MS, Bevan MJ. Cytotoxic T lymphocytes produce immune interferon in response to antigen or mitogen. J Exp Med. 1982;155(4):1198-203.

10 Scharton TM, Scott P. Natural killer cells are a source of interferon gamma that drives differentiation of CD4+ T cell subsets and induces early resistance to Leishmania major in mice. J Exp Med. 1993;178(2):567-77.

11 Sommereyns C, Paul S, Staeheli P, Michiels T. IFN-lambda (IFN- $\lambda$ ) is expressed in a tissuedependent fashion and primarily acts on epithelial cells in vivo. PLoS Pathog. 2008;4(3): e1000017.

12 Kotenko SV, Gallagher G, Baurin VV, LewisAntes A, Shen M, Shah NK, et al. IFN-lambdas mediate antiviral protection through a distinct class II cytokine receptor complex. Nat Immunol. 2003;4(1):69-77.

13 Miller JL, Anders EM. Virus-cell interactions in the induction of type 1 interferon by influenza virus in mouse spleen cells. J Gen Virol. 2003;84(Pt 1):193-202.

14 Li SF, Gong MJ, Zhao FR, Shao JJ, Xie YL, Zhang YG, et al. Type I interferons: distinct biological activities and current applications for viral infection. Cell Physiol Biochem. 2018;51(5):2377-96

15 Platanias LC. Mechanisms of type-I- and type-II-interferon-mediated signalling. Nat Rev Immunol. 2005;5(5):375-86.

16 Zhou A, Paranjape JM, Der SD, Williams BR, Silverman RH. Interferon action in triply deficient mice reveals the existence of alternative antiviral pathways. Virology. 1999;258(2): $435-40$.
17 Seo SU, Kwon HJ, Ko HJ, Byun YH, Seong BL, Uematsu S, et al. Type I interferon signaling regulates $\mathrm{Ly} 6 \mathrm{C}(\mathrm{hi})$ monocytes and neutrophils during acute viral pneumonia in mice. PLoS Pathog. 2011;7(2):e1001304.

18 Koerner I, Kochs G, Kalinke U, Weiss S, Staeheli P. Protective role of beta interferon in host defense against influenza A virus. J Virol. 2007;81(4):2025-30.

19 Davidson S, Maini MK, Wack A. Diseasepromoting effects of type I interferons in viral, bacterial, and coinfections. J Interferon Cytokine Res. 2015;35(4):252-64.

20 Bennett AL, Smith DW, Cummins MJ, Jacoby PA, Cummins JM, Beilharz MW. Low-dose oral interferon alpha as prophylaxis against viral respiratory illness: a double-blind, parallel controlled trial during an influenza pandemic year. Influenza Other Respir Viruses. 2013;7(5):854-62.

21 Steel J, Staeheli P, Mubareka S, García-Sastre A, Palese P, Lowen AC. Transmission of pandemic H1N1 influenza virus and impact of prior exposure to seasonal strains or interferon treatment. J Virol. 2010;84(1):21-6.

22 Kugel D, Kochs G, Obojes K, Roth J, Kobinger GP, Kobasa D, et al. Intranasal administration of alpha interferon reduces seasonal influenza A virus morbidity in ferrets. J Virol. 2009; 83(8):3843-51.

23 García-Sastre A, Durbin RK, Zheng H, Palese P, Gertner R, Levy DE, et al. The role of interferon in influenza virus tissue tropism. J Virol. 1998;72(11):8550-8. 
24 Price GE, Gaszewska-Mastarlarz A, Moskophidis $\mathrm{D}$. The role of alpha/beta and gamma interferons in development of immunity to influenza A virus in mice. J Virol. 2000;74(9): 3996-4003.

25 Crotta S, Davidson S, Mahlakoiv T, Desmet CJ, Buckwalter MR, Albert ML, et al. Type I and type III interferons drive redundant amplification loops to induce a transcriptional signature in influenza-infected airway epithelia. PLoS Pathog. 2013;9(11):e1003773.

26 Zanoni I, Granucci F, Broggi A. Interferon (IFN)- $\lambda$ takes the helm: immunomodulatory roles of type III IFNs. Front Immunol. 2017; $8: 1661$.

27 Galani IE, Triantafyllia V, Eleminiadou EE, Koltsida O, Stavropoulos A, Manioudaki M, et al. Interferon- $\lambda$ mediates non-redundant front-line antiviral protection against influenza virus infection without compromising host fitness. Immunity. 2017;46(5):875-890. e6.

28 Klinkhammer J, Schnepf D, Ye L, Schwaderlapp M, Gad HH, Hartmann R, et al. IFN- $\lambda$ prevents influenza virus spread from the upper airways to the lungs and limits virus transmission. Elife. 2018;7:e33354.

29 Wilson EB, Yamada DH, Elsaesser H, Herskovitz J, Deng J, Cheng G, et al. Blockade of chronic type I interferon signaling to control persistent LCMV infection. Science. 2013; 340(6129):202-7.

30 Teijaro JR, Ng C, Lee AM, Sullivan BM, Sheehan KC, Welch M, et al. Persistent LCMV infection is controlled by blockade of type I interferon signaling. Science. 2013;340(6129): 207-11.

31 La Gruta NL, Kedzierska K, Stambas J, Doherty PC. A question of self-preservation: immunopathology in influenza virus infection. Immunol Cell Biol. 2007;85(2):85-92.

32 Tumpey TM, García-Sastre A, Taubenberger JK, Palese P, Swayne DE, Pantin-Jackwood MJ, et al. Pathogenicity of influenza viruses with genes from the 1918 pandemic virus: functional roles of alveolar macrophages and neutrophils in limiting virus replication and mortality in mice. J Virol. 2005;79(23):1493344.

33 Cillóniz C, Shinya K, Peng X, Korth MJ, Proll $\mathrm{SC}$, Aicher LD, et al. Lethal influenza virus infection in macaques is associated with early dysregulation of inflammatory related genes. PLoS Pathog. 2009;5(10):e1000604.

34 Kobasa D, Jones SM, Shinya K, Kash JC, Copps J, Ebihara H, et al. Aberrant innate immune response in lethal infection of macaques with the 1918 influenza virus. Nature. 2007;445(7125):319-23.

35 Cheung CY, Poon LL, Lau AS, Luk W, Lau YL, Shortridge KF, et al. Induction of proinflammatory cytokines in human macrophages by influenza A (H5N1) viruses: a mechanism for the unusual severity of human disease? Lancet. 2002;360(9348):1831-7.
36 Hayden FG, Fritz R, Lobo MC, Alvord W, Strober W, Straus SE. Local and systemic cytokine responses during experimental human influenza A virus infection. Relation to symptom formation and host defense. J Clin Invest. 1998;101(3):643-9.

37 Kaiser L, Fritz RS, Straus SE, Gubareva L, Hayden FG. Symptom pathogenesis during acute influenza: interleukin- 6 and other cytokine responses. J Med Virol. 2001;64(3):2628

38 Herold S, Becker C, Ridge KM, Budinger GR Influenza virus-induced lung injury: pathogenesis and implications for treatment. Eur Respir J. 2015;45(5):1463-78.

39 Högner K, Wolff T, Pleschka S, Plog S, Gruber AD, Kalinke U, et al. Macrophage-expressed IFN- $\beta$ contributes to apoptotic alveolar epithelial cell injury in severe influenza virus pneumonia. PLoS Pathog.2013;9(2):e1003188.

40 Herold S, Steinmueller M, von Wulffen W, Cakarova L, Pinto R, Pleschka S, et al. Lung epithelial apoptosis in influenza virus pneumonia: the role of macrophage-expressed TNF-related apoptosis-inducing ligand. J Exp Med. 2008;205(13):3065-77.

41 Chaperot L, Blum A, Manches O, Lui G, Angel J, Molens JP, et al. Virus or TLR agonists induce TRAIL-mediated cytotoxic activity of plasmacytoid dendritic cells. J Immunol. 2006;176(1):248-55

42 Davidson S, Crotta S, McCabe TM, Wack A. Pathogenic potential of interferon $\alpha \beta$ in acute influenza infection. Nat Commun. 2014;5: 3864.

43 Fujikura D, Chiba S, Muramatsu D, Kazumata M, Nakayama Y, Kawai T, et al. Type-I interferon is critical for FasL expression on lung cells to determine the severity of influenza. PLoS One. 2013;8(2):e55321.

44 Iskander M, Booy R, Lambert S. The burden of influenza in children. Curr Opin Infect Dis. 2007;20(3):259-63.

45 Cowling BJ, Chan KH, Fang VJ, Lau LLH, So HC, Fung ROP, et al. Comparative epidemiology of pandemic and seasonal influenza A in households. NEnglJMed.2010;362(23):217584.

46 Zinkernagel RM. Immunological memory $\neq$ protective immunity. Cell Mol Life Sci. 2012; 69(10):1635-40.

47 Loeb M, Singh PK, Fox J, Russell ML, Pabbaraju K, Zarra D, et al. Longitudinal study of influenza molecular viral shedding in Hutterite communities. J Infect Dis. 2012;206(7): 1078-84.

48 Coates BM, Staricha KL, Koch CM, Cheng Y, Shumaker DK, Budinger GRS, et al. Inflammatory monocytes drive influenza A virusmediated lung injury in juvenile mice. J Immunol. 2018;200(7):2391-404.

49 Oshansky CM, Gartland AJ, Wong SS, Jeevan T, Wang D, Roddam PL, et al. Mucosal immune responses predict clinical outcomes during influenza infection independently of age and viral load. Am J Respir Crit Care Med. 2014;189(4):449-62.
50 Porritt RA, Hertzog PJ. Dynamic control of type I IFN signalling by an integrated network of negative regulators. Trends Immunol. 2015;36(3):150-60.

51 Lee AJ, Ashkar AA. The dual nature of type I and type II interferons. Front Immunol. 2018; 9:2061.

52 Moore BR, Krakowka S, Cummins JM, Robertson JT. Changes in airway inflammatory cell populations in standardbred racehorses after interferon-alpha administration. Vet Immunol Immunopathol. 1996;49(4):34758.

53 Gibb DR, Liu J, Natarajan P, Santhanakrishnan M, Madrid DJ, Eisenbarth SC, et al. Type I IFN is necessary and sufficient for inflammation-induced red blood cell alloimmunization in mice. J Immunol. 2017;199(3):104150.

54 Kudo D, Uno K, Aoyagi T, Akahori Y, Ishii K, Kanno E, et al. Low-dose interferon- $a$ treatment improves survival and inflammatory responses in a mouse model of fulminant acute respiratory distress syndrome. Inflammation. 2013;36(4):812-20.

55 Gold JA, Hoshino Y, Jones MB, Hoshino S, Nolan A, Weiden MD. Exogenous interferonalpha and interferon-gamma increase lethality of murine inhalational anthrax. PLoS One. 2007;2(8):e736.

56 Kurche JS, Haluszczak C, McWilliams JA, Sanchez PJ, Kedl RM. Type I IFN-dependent $\mathrm{T}$ cell activation is mediated by IFN-dependent dendritic cell OX40 ligand expression and is independent of T cell IFNR expression. J Immunol. 2012;188(2):585-93.

57 Santini SM, Lapenta C, Logozzi M, Parlato S, Spada M, Di Pucchio T, et al. Type I interferon as a powerful adjuvant for monocytederived dendritic cell development and activity in vitro and in Hu-PBL-SCID mice. J Exp Med. 2000;191(10):1777-88.

58 Montoya M, Schiavoni G, Mattei F, Gresser I, Belardelli F, Borrow P, et al. Type I interferons produced by dendritic cells promote their phenotypic and functional activation. Blood. 2002;99(9):3263-71.

59 Simmons DP, Wearsch PA, Canaday DH, Meyerson HJ, Liu YC, Wang Y, et al. Type I IFN drives a distinctive dendritic cell maturation phenotype that allows continued class II MHC synthesis and antigen processing. J Immunol. 2012;188(7):3116-26.

60 Downes JE, Marshall-Clarke S. Innate immune stimuli modulate bone marrow-derived dendritic cell production in vitro by tolllike receptor-dependent and -independent mechanisms. Immunology. 2010;131(4):51324.

61 Rudd BD, Luker GD, Luker KE, Peebles RS, Lukacs NW. Type I interferon regulates respiratory virus infected dendritic cell maturation and cytokine production. Viral Immunol, 2007;20(4):531-40. 
62 Moltedo B, Li W, Yount JS, Moran TM. Unique type I interferon responses determine the functional fate of migratory lung dendritic cells during influenza virus infection. PLoS Pathog. 2011;7(11):e1002345.

63 Helft J, Manicassamy B, Guermonprez P, Hashimoto D, Silvin A, Agudo J, et al. Crosspresenting $\mathrm{CD} 103+$ dendritic cells are protected from influenza virus infection. J Clin Invest. 2012;122(11):4037-47.

64 Langlois RA, Varble A, Chua MA, GarcíaSastre A, tenOever BR. Hematopoietic-specific targeting of influenza A virus reveals replication requirements for induction of antiviral immune responses. Proc Natl Acad Sci U S A. 2012;109(30):12117-22.

65 Hemann EA, Green R, Turnbull JB, Langlois RA, Savan R, Gale M Jr. Interferon- $\lambda$ modulates dendritic cells to facilitate $\mathrm{T}$ cell immunity during infection with influenza $\mathrm{A}$ virus. Nat Immunol. 2019;20(8):1035-45.

66 Brandes M, Klauschen F, Kuchen S, Germain RN. A systems analysis identifies a feedforward inflammatory circuit leading to lethal influenza infection. Cell. 2013;154(1):197-212.

67 Stifter SA, Bhattacharyya N, Pillay R, Flórido M, Triccas JA, Britton WJ, et al. Functional interplay between type I and II interferons is essential to limit influenza A virus-induced tissue inflammation. PLoS Pathog. 2016;12(1): e1005378.

68 Julkunen I, Melén K, Nyqvist M, Pirhonen J, Sareneva T, Matikainen S. Inflammatory responses in influenza A virus infection. Vaccine. 2000;19(Suppl 1):S32-7.

69 Schultz-Cherry S. Role of NK cells in influenza infection. Curr Top Microbiol Immunol. 2015;386:109-20.

70 Biron CA, Nguyen KB, Pien GC, Cousens LP, Salazar-Mather TP. Natural killer cells in antiviral defense: function and regulation by innate cytokines. Annu Rev Immunol. 1999; 17:189-220.

71 Nguyen KB, Salazar-Mather TP, Dalod MY, Van Deusen JB, Wei XQ, Liew FY, et al. Coordinated and distinct roles for IFN-alpha beta, IL-12, and IL-15 regulation of NK cell responses to viral infection. J Immunol. 2002; 169(8):4279-87.

72 Hwang I, Scott JM, Kakarla T, Duriancik DM, Choi S, Cho C, et al. Activation mechanisms of natural killer cells during influenza virus infection. PLoS One. 2012;7(12):e51858.

73 Arimori Y, Nakamura R, Yamada H, Shibata K, Maeda N, Kase T, et al. Type I interferon plays opposing roles in cytotoxicity and interferon- $\gamma$ production by natural killer and CD8 T cells after influenza A virus infection in mice. J Innate Immun. 2014;6(4):456-66.

74 Havenar-Daughton C, Kolumam GA, Murali-Krishna K. Cutting edge: the direct action of type I IFN on CD4 T cells is critical for sustaining clonal expansion in response to a viral but not a bacterial infection. J Immunol. 2006; 176(6):3315-9.
75 Kolumam GA, Thomas S, Thompson LJ, Sprent J, Murali-Krishna K. Type I interferons act directly on CD8 T cells to allow clonal expansion and memory formation in response to viral infection. J Exp Med. 2005; 202(5):637-50.

76 Thompson LJ, Kolumam GA, Thomas S, Murali-Krishna $\mathrm{K}$. Innate inflammatory signals induced by various pathogens differentially dictate the IFN-I dependence of CD8 T cells for clonal expansion and memory formation. I Immunol. 2006;177(3):1746-54

77 Welsh RM, Bahl K, Marshall HD, Urban SL. Type 1 interferons and antiviral CD8 T-cell responses. PLoS Pathog. 2012;8(1):e1002352.

78 Duerr CU, McCarthy CD, Mindt BC, Rubio M, Meli AP, Pothlichet J, et al. Type I interferon restricts type 2 immunopathology through the regulation of group 2 innate lymphoid cells. Nat Immunol. 2016;17(1):65-75.

79 Garcin G, Bordat Y, Chuchana P, Monneron D, Law HK, Piehler J, et al. Differential activity of type I interferon subtypes for dendritic cell differentiation. PLoS One. 2013;8(3): e58465.

80 James CM, Abdad MY, Mansfield JP, Jacobsen HK, Vind AR, Stumbles PA, et al. Differential activities of alpha/beta IFN subtypes against influenza virus in vivo and enhancement of specific immune responses in DNA vaccinated mice expressing haemagglutinin and nucleoprotein. Vaccine. 2007;25(10): 1856-67.

81 Marié I, Durbin JE, Levy DE. Differential viral induction of distinct interferon-alpha genes by positive feedback through interferon regulatory factor-7. EMBO J. 1998;17(22):6660-9.

82 Juang YT, Lowther W, Kellum M, Au WC, Lin R, Hiscott J, et al. Primary activation of interferon $\mathrm{A}$ and interferon $\mathrm{B}$ gene transcription by interferon regulatory factor 3 . Proc Natl Acad Sci U S A. 1998;95(17):9837-42.

83 Samuelsson CV, Lienenklaus S, Müller PP, Zawatzky R, Hauser H, Weiss S. Transformation of mouse fibroblasts alters the induction pattern of type I IFNs after virus infection. Biochem Biophys Res Commun. 2005;335(2): 584-9.

84 Taniguchi T, Takaoka A. A weak signal for strong responses: interferon-alpha/beta revisited. Nat Rev Mol Cell Biol. 2001;2(5):378-86.

85 Kawai T, Akira S. The role of pattern-recognition receptors in innate immunity: update on Toll-like receptors. Nat Immunol. 2010; 11(5):373-84.

86 Deonarain R, Alcamí A, Alexiou M, Dallman MJ, Gewert DR, Porter AC. Impaired antiviral response and alpha/beta interferon induction in mice lacking beta interferon. J Virol. 2000;74(7):3404-9.

87 Jewell NA, Vaghefi N, Mertz SE, Akter P, Peebles RS Jr, Bakaletz LO, et al. Differential type I interferon induction by respiratory syncytial virus and influenza a virus in vivo. J Virol. 2007;81(18):9790-800.
88 de Weerd NA, Vivian JP, Nguyen TK, Mangan NE, Gould JA, Braniff SJ, et al. Structural basis of a unique interferon- $\beta$ signaling axis mediated via the receptor IFNAR1. Nat Immunol. 2013;14(9):901-7.

89 Jaitin DA, Roisman LC, Jaks E, Gavutis M, Piehler J, Van der Heyden J, et al. Inquiring into the differential action of interferons (IFNs): an IFN-alpha2 mutant with enhanced affinity to IFNAR1 is functionally similar to IFN-beta. Mol Cell Biol. 2006; 26(5):1888-97.

90 Saraiva M, O'Garra A. The regulation of IL10 production by immune cells. Nat Rev Immunol. 2010;10(3):170-81.

91 Sharpe AH, Wherry EJ, Ahmed R, Freeman GJ. The function of programmed cell death 1 and its ligands in regulating autoimmunity and infection. Nat Immunol. 2007;8(3):23945.

92 Thomas C, Moraga I, Levin D, Krutzik PO, Podoplelova Y, Trejo A, et al. Structural linkage between ligand discrimination and receptor activation by type I interferons. Cell. 2011;146(4):621-32.

93 Levin D, Harari D, Schreiber G. Stochastic receptor expression determines cell fate upon interferon treatment. Mol Cell Biol. 2011;31(16):3252-66.

94 Levin D, Schneider WM, Hoffmann $\mathrm{HH}$ Yarden G, Busetto AG, Manor O, et al. Multifaceted activities of type I interferon are revealed by a receptor antagonist. Sci Signal. 2014;7(327):ra50.

95 Kalie E, Jaitin DA, Abramovich R, Schreiber G. An interferon alpha2 mutant optimized by phage display for IFNAR1 binding confers specifically enhanced antitumor activities. J Biol Chem. 2007;282(15):11602-11.

96 Jaks E, Gavutis M, Uzé G, Martal J, Piehler J. Differential receptor subunit affinities of type I interferons govern differential signal activation. J Mol Biol. 2007;366(2):525-39.

97 Shepardson KM, Larson K, Johns LL, Stanek $\mathrm{K}$, Cho H, Wellham J, et al. IFNAR2 is required for anti-influenza immunity and alters susceptibility to post-influenza bacterial superinfections. Front Immunol. 2018;9: 2589.

98 Schreiber G. The molecular basis for differential type I interferon signaling. J Biol Chem. 2017;292(18):7285-94.

99 Davis MM, Taubert K, Benin AL, Brown DW, Mensah GA, Baddour LM, et al. Influenza vaccination as secondary prevention for cardiovascular disease: a science advisory from the American Heart Association/ American College of Cardiology. J Am Coll Cardiol. 2006;48(7):1498-502.

100 Biondo C, Lentini G, Beninati C, Teti G. The dual role of innate immunity during influenza. Biomed J. 2019;42(1):8-18. 